\title{
Effective Charge on Acetylcholinesterase Active Sites Determined from the Ionic Strength Dependence of Association Rate Constants with Cationic Ligands ${ }^{\dagger}$
}

\author{
Hans-Jürgen Nolte, Terrone L. Rosenberry,* and Eberhard Neumann
}

\begin{abstract}
The reaction of the specific fluorescent cationic ligand $N$-methylacridinium with the active site of $11 \mathrm{~S}$ acetylcholinesterase from electric eel was monitored by temperature-jump relaxation kinetics at a variety of ionic strengths. The ionic strength dependence of the bimolecular association rate constant is analyzed with a Brфnsted-Debye-Hückel expression and leads to estimates of the association rate constant at zero ionic strength of $k_{12}{ }^{0}=1.1 \times 10^{10} \mathrm{M}^{-1} \mathrm{~s}^{-1}$ at 25 ${ }^{\circ} \mathrm{C}$ and the net charge number of the enzyme active site of $Z_{\mathrm{E}}=-6.3$. The ionic strength dependence of the second-order hydrolysis rate constant $k_{\text {cat }} / K_{\text {app }}$ for acetylthiocholine under steady-state conditions is also very pronounced and indicates a value of $Z_{\mathrm{E}}=-9$. Thus, a large effective negative charge on the enzyme active site appears to be a general characteristic
\end{abstract}

\begin{abstract}
A cetylcholinesterase is an extremely efficient catalyst of acetylcholine hydrolysis at neuromuscular junctions [see, for example, Neumann et al. (1978) and Rosenberry (1979)]. This efficiency reflects both a very high first-order hydrolysis rate constant of $k_{\text {cat }}=1.6 \times 10^{4} \mathrm{~s}^{-1}$ at high substrate concentrations and a very high second-order constant of $k_{\text {cat }} / K_{\text {app }}$ $=2 \times 10^{8} \mathrm{M}^{-1} \mathrm{~s}^{-1}$ at lower substrate concentrations or under conditions of excess enzyme [Rosenberry, 1975a; values at 25 ${ }^{\circ} \mathrm{C}$, ionic strength $\left(I_{\mathrm{c}}\right)$ of $\left.0.1 \mathrm{M}\right]$. Recently, we investigated the basis of this high second-order rate constant by measuring the bimolecular association rate constant $k_{12}$ for the reaction of the fluorescent ligand $\mathrm{N}$-methylacridinium with acetylcholinesterase from the electric eel Electrophorus electricus (Rosenberry \& Neumann, 1977). This cationic ligand binds with high specificity to each of the four independent sites in the 11 S enzyme tetramer (Mooser \& Sigman, 1974). Temperature-jump relaxation kinetics showed that $k_{12}=1.2 \times 10^{9}$ $\mathrm{M}^{-1} \mathrm{~s}^{-1}\left(23^{\circ} \mathrm{C}, \mathrm{pH} 8, I_{\mathrm{c}}=0.13 \mathrm{M}\right)$, an unusually high value for an enzyme-ligand interaction.

In this study we have pursued the physical basis for this high $k_{12}$ value by examining its ionic strength dependence. Previous studies have demonstrated the dramatic effect of ionic strength on cationic ligand affinities for acetylcholinesterase from Electrophorus (Mooser \& Sigman, 1974), from the torpedo Torpedo marmorata (Changeux, 1966), and from erythrocytes (Pyttel \& Robinson, 1974). Our report confirms this effect on equilibrium affinities and extends the analysis to individual association and dissociation rate constants to provide a

\footnotetext{
${ }^{\dagger}$ From the Max-Planck-Institut für Biochemie, D-8033 Martinsried, Federal Republic of Germany (H.-J.N. and E.N.), and the Departments of Biochemistry and Neurology, College of Physicians and Surgeons, Columbia University, New York, New York 10032 (T.L.R.). Received September 19, 1979. Supported in part by National Science Foundation Grant PCM77 09383, National Institutes of Health Grants NS-03304 and NS-11766, and Deutsche Forschungsgemeinschaft Grant NE 227/1.

* Correspondence should be addressed to this author at the Department of Pharmacology, Case Western Reserve University, Cleveland, $\mathrm{OH}$ 44106.
}

of its interaction with cationic ligands. The ionic strength dependence of $k_{\mathrm{cat}} / K_{\mathrm{app}}$ is identical with that of sodium chloride, sodium phosphate, and sodium citrate, thus ruling out any possibility that the phenomena arise from a specific, partially competitive binding of $\mathrm{Na}^{+}$to the enzyme active site. Substitution of the calculated electrostatic parameters into theoretical equations indicates that the most significant effect of these $Z_{\mathrm{E}}$ values is a 2-3 order of magnitude reduction in the rate constant for dissociation of the initial ligand-enzyme encounter complex; this decrease renders the bimolecular reaction diffusion controlled. The high value of $k_{12}{ }^{0}$ and the space requirements of six to nine charged groups suggest that regions of the enzyme surface area larger than the catalytic sites themselves are effective in trapping cationic ligands.

mechanistic basis for the ionic strength dependence. A high net negative charge on the enzyme active site appears to be responsible for making the association reaction primarily diffusion controlled. The isoelectric point $(\mathrm{p} I)$ of the eel enzyme is 4.5 (Chen et al., 1974). Thus, at $\mathrm{pH} 7$ anionic groups dominate the surface charge and several of these groups appear to influence the association reaction at each catalytic site. Similar electrostatic features govern the interaction of both $N$-methylacridinium and the acetylcholine analogue acetylthiocholine with the enzyme active site.

\section{Experimental Section}

Materials. Acetylcholinesterase from the electric organs of the eel E. electricus was an $11 \mathrm{~S}$ preparation. Its specific activity corresponded to $\sim 55 \%$ of the maximal activity reported for 11S acetylcholinesterase (Rosenberry, 1975a), and its normality was determined from equilibrium and kinetic titrations with $N$-methylacridinium. The concentration of recrystallized $N$-methylacridinium perchlorate in solutions was determined from $\epsilon_{358}=21300 \mathrm{M}^{-1} \mathrm{~cm}^{-1}$ (Rosenberry \& Neumann, 1977).

Buffer solutions contained defined mixtures of sodium monobasic and dibasic phosphates, and other salts were omitted except where noted to avoid the quenching of $N$ methylacridinium fluorescence (Rosenberry \& Neumann, 1977). The solution ionic strengths $\left(I_{c}\right)$ were calculated by assuming complete dissociation of $\mathrm{NaH}_{2} \mathrm{PO}_{4}$ and partial dissociation of $\mathrm{Na}_{2} \mathrm{HPO}_{4}$ according to the equilibrium $\left[\mathrm{NaHPO}_{4}^{-}\right] /\left(\left[\mathrm{Na}^{+}\right]\left[\mathrm{HPO}_{4}{ }^{2-}\right]\right)=4.0 \mathrm{M}^{-1}$ (Smith \& Alberty, 1956). The ionic strength of sodium citrate was calculated by assuming $\mathrm{p} K_{\mathrm{a} 3}=6.39$ (Bates \& Pinching, 1949).

Fluorescence Measurements. Fluorescence data were collected in a temperature-jump apparatus similar to that described previously (Rigler et al., 1974; Rosenberry \& Neumann, 1977). The temperature increase was triggered by a 20 - or $30-\mathrm{kV}$ discharge from a $1 \times 10^{-8}, 2 \times 10^{-8}$, or $5 \times$ $10^{-8} \mathrm{~F}$ capacitor through the $0.8-\mathrm{mL}$ sample compartment, but the final temperature in all cases was adjusted to $25.0^{\circ} \mathrm{C}$. 
Scheme 1

$$
\mathrm{E}+\mathrm{L} \underset{k_{21}}{\stackrel{k_{12}}{\rightleftharpoons}} \mathrm{EL}
$$

Scheme II

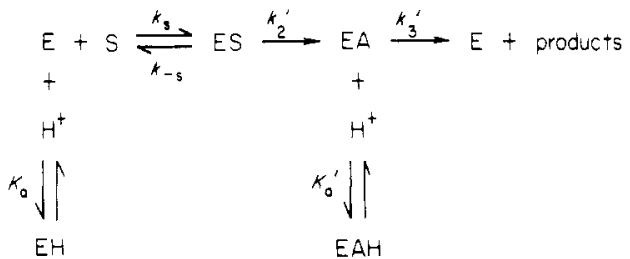

The fluorescence relaxation trace was stored on a Bruker transient recorder, and relaxation times and amplitudes were obtained by superpositioning the stored trace upon a calibrated multiple exponential function [see Rosenberry \& Neumann (1977)] with a dual-beam oscilloscope. Usually three to four temperature-jump measurements per titration step were sufficient to assure good reproducibility and each titration involved 8 to $13 C_{\mathrm{L}}^{\text {tot }}$ points

Steady-State Acetylcholinesterase Assay. Enzyme activity toward acetylthiocholine was measured with a modification of the spectrophotometric assay of Ellman et al. (1961) at 25 ${ }^{\circ} \mathrm{C}$. The concentration of 5,5'-dithiobis(2-nitrobenzoic acid) was fixed at $0.33 \mathrm{mM}$, but the phosphate buffer $I_{\mathrm{c}}$ was varied between 2.2 and $250 \mathrm{mM}$, the acetylthiocholine concentration ranged from 1.6 to $25 \mu \mathrm{M}$, and the $\mathrm{pH}$ was adjusted between 5.9 and 8.2 . Small corrections $(<10 \%)$ were applied to rates measured at the lowest $\mathrm{pH}$ values to compensate for slight decreases in the absorbance of reduced 5,5'-dithiobis(2nitrobenzoate). Typical rates with $3.3 \mu \mathrm{M}$ acetylthiocholine were measured at $412 \mathrm{~nm}$ on the 0.05 -A full-scale setting of a Perkin-Elmer 572 spectrophotometer. Final rates were triplicate averages randomized with respect to the independent variable, usually ionic strength.

Ligand Interaction Schemes. The binding of the fluorescent ligand $N$-methylacridinium to acetylcholinesterase active sites was analyzed according to the simple, one-step process indicated in Scheme I. The association rate constant $k_{12}$ and the dissociation rate constant $k_{21}$ define the equilibrium dissociation constant $K_{\mathrm{D}} \equiv k_{21} / k_{12}$. Inclusion of an independent additional binding site for the ligand in enzyme preparations [Scheme II' in Rosenberry \& Neumann (1977)] was considered during analysis of some experiments but was rejected due to insufficient evidence (see Results).

The steady-state hydrolysis of acetylthiocholine by acetylcholinesterase was interpreted according to the mechanism in Scheme II. In Scheme II the substrate $\mathrm{S}$ combines with the free enzyme $\mathrm{E}$ and the complex ES is converted to an intermediate acetylenzyme EA [see Rosenberry (1975a)]. A proton with dissociation constant $K_{\mathrm{a}}$ competes with cationic substrates for the binding site in $\mathrm{E}$, and a proton with dissociation constant $K_{\mathrm{a}}^{\prime}$ blocks deacetylation of EA (Krupka, 1966; Rosenberry, $1975 \mathrm{~b}$ ). The rate equation involving the hydrolysis rate $v$ that corresponds to Scheme II is given by

$$
\frac{1}{v}=\frac{1}{k_{\mathrm{cat}} C_{\mathrm{E}}{ }^{\mathrm{tot}}}\left[1+\frac{k_{\mathrm{cat}} C_{\mathrm{H}^{+}}}{k_{3}{ }^{\prime} K_{\mathrm{a}}{ }^{\prime}}+\frac{K_{\mathrm{app}}}{C_{\mathrm{S}}}\left(1+\frac{C_{\mathrm{H}^{+}}}{K_{\mathrm{a}}}\right)\right]
$$

where $C_{\mathrm{X}}$ is the concentration of species $\mathrm{X}, C_{\mathrm{E}}{ }^{\text {tot }}$ is the total enzyme concentration, $k_{\text {cat }}=k_{2}^{\prime} k_{3}^{\prime} /\left(k_{2}^{\prime}+k_{3}^{\prime}\right)$, and $K_{\text {app }}=$ $k_{\text {cat }}\left(k_{-\mathrm{s}}+k_{2}^{\prime}\right) /\left(k_{\mathrm{s}} k_{2}^{\prime}\right)$ [see Rosenberry (1975a)].

Relaxation Data. The expression relating the observed relaxation time $\tau$ to the intrinsic rate constants in Scheme I
(Eigen \& DeMaeyer, 1963) is given by

$$
\tau^{-1}=k_{12}\left(\bar{C}_{\mathrm{E}}+\bar{C}_{\mathrm{L}}\right)+k_{21}
$$

where $\bar{C}_{\mathrm{E}}$ and $\bar{C}_{\mathrm{L}}$ are the respective equilibrium concentrations of enzyme active sites and of ligand. An alternative expression (Rosenberry \& Neumann, 1977) that involves only total concentrations $C_{\mathrm{E}}{ }^{\text {tot }}$ and $C_{\mathrm{L}}$ tot (i.e., in Scheme I $C_{\mathrm{E}}{ }^{\text {tot }}=\bar{C}_{\mathrm{E}}+$ $\left.\bar{C}_{\mathrm{EL}}\right)$ is given by eq 3 and is used here.

$$
\begin{aligned}
& \tau^{-2}=k_{12}{ }^{2}\left(K_{\mathrm{D}}+C_{\mathrm{E}}{ }^{\mathrm{tot}}\right)^{2}+2 k_{12}{ }^{2}\left(K_{\mathrm{D}}-C_{\mathrm{E}}{ }^{\mathrm{tot}}\right) C_{\mathrm{L}}^{\text {tot }}+ \\
& k_{12}^{2}\left(C_{\mathrm{L}}{ }^{\text {tot }}\right)^{2}
\end{aligned}
$$

When $C_{\mathrm{E}}{ }^{\text {tot }}$ is constant during a progressive titration with the ligand, eq 3 is a second-order polynomial in $C_{\mathrm{L}}$ tot ;etermination of the coefficients of the polynomial by regression analysis allows simultaneous evaluation of $k_{12}, C_{\mathrm{E}}{ }^{\text {tot }}$, and $K_{\mathrm{D}}$. Slight corrections for enzyme dilution during the titration can be introduced (Rosenberry \& Neumann, 1977) that permit calculation of the initial value of $C_{\mathrm{E}}^{\text {tot }} \equiv C_{\mathrm{E}}$ init. Enzyme dilution arises from the progressive addition of stock ligand solution but is partially compensated for by evaporation accompanying deaeration of the sample after each ligand addition. An average dilution factor was calculated from the known volume of ligand solution added (generally $0.6-2.5 \%$ of the total sample volume) and the average incremental volume loss due to evaporation as measured by the increased conductivity of the sample (generally $0.3-1.3 \%$ ).

In experiments where two observed relaxation steps suggested the presence of a second independent binding site for ligand, an analysis of relaxation amplitudes was conducted to provide quantitative estimates of the coupling factor $\theta$ between the two relaxation steps (Rosenberry \& Neumann, 1977). If coupling is negligible, $\theta$ increases to a value of 1 . Values of $\theta$ less than 1 can also be detected by analysis of relaxation times according to eq 2 . When coupling is significant, the slower relaxation time is given by an expression analogous to eq 2 in which $\bar{C}_{\mathrm{E}}$ is replaced by $\bar{C}_{\mathrm{E}} \theta$ (Rosenberry \& Neumann, 1977). Thus, deviation of $\tau^{-1}$ values at low $\bar{C}_{\mathrm{L}}$ from the linear relationship predicted by eq 2 is an indication of a possible coupling factor $\theta$.

Analysis of equilibrium fluorescence data was carried out as previously described (Rosenberry \& Neumann, 1977).

Steady-State Data. Measurements of $K_{\mathrm{a}}$ were made at low acetylthiocholine concentrations $\left(C_{\mathrm{S}}<K_{\mathrm{app}}\right)$ by determining the ratio $R$ for $v_{1}$ at low $\mathrm{pH}_{1}$ to $v_{2}$ at high $\mathrm{pH}_{2}$ at a fixed $I_{\mathrm{c}}$. Calculated values of $K_{\mathrm{a}}$ were obtained from $R$ according to

$$
K_{\mathrm{a}}=\frac{R 10^{-\mathrm{pH}_{1}}-10^{-\mathrm{pH}_{2}}}{1-R}
$$

Equation 4 assumes that $K_{\mathrm{a}} \simeq k_{3}{ }^{\prime} K_{\mathrm{a}}{ }^{\prime} / k_{\text {cat }}$. Second-order hydrolysis rate constants $k_{\text {cat }} / K_{\text {app }}$ were calculated from pseudo-first-order rate constants $k_{\text {cat }} C_{\mathrm{E}}^{\text {tot }} / K_{\text {app }}$ by assuming $k_{\text {cat }} / K_{\text {app }}=2.5 \times 10^{8} \mathrm{M}^{-1} \mathrm{~s}^{-1}$ at $I_{\mathrm{c}}=0.10 \mathrm{M}$ and $\mathrm{pH} 7.5$ (Hillman \& Mautner, 1970; Rosenberry, 1975a). Estimates of $k_{\text {cat }} C_{\mathrm{E}}^{\text {tot }} / K_{\text {app }}$ were obtained at $C_{\mathrm{S}}<K_{\text {app }}$ by rearrangement of eq 1 according to

$$
\frac{k_{\mathrm{cat}} C_{\mathrm{E}}^{\mathrm{tot}}}{K_{\mathrm{app}}} \simeq \frac{v}{C_{\mathrm{S}}}\left(1+\frac{C_{\mathrm{S}}}{K_{\mathrm{app}}}\right)\left(1+\frac{C_{\mathrm{H}^{+}}}{K_{\mathrm{a}}}\right)
$$

Equation 5 also assumes that $K_{\mathrm{a}} \simeq k_{3}{ }^{\prime} K_{\mathrm{a}}{ }^{\prime} / k_{\text {cat }}$. This assumption is equivalent to considering the "apparent $K_{\text {app " }}$ (i.e., the negative abscissa intercept of a plot of $v^{-1}$ vs. $C_{\mathrm{S}}{ }^{-1}$ according to eq 1) to be independent of $\mathrm{pH}$. For acetylcholine hydrolysis at $I_{\mathrm{c}}=0.1 \mathrm{M}$, the assumption appears valid: $\mathrm{p} K_{\mathrm{a}}=6.3$, $k_{3}{ }^{\prime} / k_{\text {cat }} \simeq 1$, and $\mathrm{p} K_{\mathrm{a}}{ }^{\prime}=6.5$ (Rosenberry, 1975a,b); furthermore, the apparent $K_{\text {app }}$ appears to be independent of pH 
at various $I_{\mathfrak{c}}$ values (H. R. Smissaert, unpublished results). Thus, the assumption appears reasonable for acetylthiocholine hydrolysis. Moreover, eq 4 and 5 were utilized here only at low $C_{\mathrm{S}}$ (except for the lowest $I_{\mathrm{c}}$ investigated, $C_{\mathrm{S}} / K_{\text {app }}<0.25$ ), a condition under which $K_{\mathrm{a}}$ and $k_{\text {cat }} C_{\mathrm{E}}{ }^{\text {tot }} / K_{\text {app }}$ estimates are little affected even by moderate differences between $K_{\mathrm{a}}$ and $k_{3}{ }^{\prime} K_{\mathrm{a}}{ }^{\prime} / k_{\text {cat }}$.

Theoretical Treatment of Ionic Strength Dependence. Analysis of the dependence of observed rate constants on ionic strength was based on concepts introduced by Bronsted and Bjerrum as developed by Scatchard (1932). The association rate constant $k_{12}$ in Scheme I thus is given by

$$
k_{12}=k_{12}{ }^{0} f_{\mathrm{E}} f_{\mathrm{L}} / f^{*}
$$

where $f_{\mathrm{E}}$ and $f_{\mathrm{L}}$ are activity coefficients of $\mathrm{E}$ and $\mathrm{L}, f^{*}$ is the activity coefficient for the activated complex formed on the reaction pathway, and $k_{12}{ }^{0}$ is the association rate constant at zero ionic strength where all $f$ terms equal 1. Application of the Debye-Hückel theory to the calculation of the activity coefficients [e.g., see Kortüm (1972)] leads to

$$
\log k_{12}=\log k_{12}{ }^{0}+\frac{2 A Z_{\mathrm{E}} Z_{\mathrm{L}} I_{\mathrm{c}}^{1 / 2}}{1+B \bar{a} I_{\mathrm{c}}{ }^{1 / 2}}
$$

In eq 7, $A$ and $B$ are constants (for $25^{\circ} \mathrm{C}$ and the dielectric constant of water $\epsilon=79, A=0.509 \mathrm{M}^{-1 / 2}$ and $B=3.29 \mathrm{M}^{-1 / 2}$ $\mathrm{nm}^{-1}$ ); the ionic strength $I_{\mathrm{c}}=0.5 \sum_{\mathrm{i}} Z_{\mathrm{i}}^{2} C_{\mathrm{i}}$, where $Z_{\mathrm{i}}$ and $C_{\mathrm{i}}$ are the charge number and concentration of species $\mathrm{i} ; \bar{a}$ is the mean distance of closest approach between $E$ and counterions. In this study, $Z_{\mathrm{L}}=+1$ for all ligands.

The application of eq 6 and 7 to a diffusion-controlled reaction involves certain approximations. For example, the equilibrium condition for the ionic atmospheres appears to be unfulfilled for ionic diffusional encounter complexes (Eigen, 1954; Eigen et al., 1964). It must be assumed that the relatively high concentrations of inert strong electrolytes in these experiments provide sufficient electrostatic screening to permit estimation of the activity coefficients for the nonequilibrium states during the relaxation in the same formal way as for equilibrated ionic atmospheres. A second approximation involves the solution of the Poisson-Boltzmann equation used in the derivation of eq 7. The solution generally utilizes the assumption that $Z_{\mathrm{L}} e_{0} \bar{\psi}_{\mathrm{E}}(r) \ll k T$, where $\bar{\psi}_{\mathrm{E}}(r)$ is the mean electrostatic potential at a distance $r$ from the enzyme active site (see the Appendix), but this assumption does not appear justified, particularly with the large $Z_{E}$ estimates obtained here. An alternative solution which avoids this assumption has been developed by Pierce [see Rice \& Nagasawa (1961)]. The solution to the electrostatic potential is expressed as a power series, the first term of which is equivalent to the corresponding expression in the Debye-Hückel approximation. When this method is applied to our case with the parameter values collected in Table III, it is shown that the Debye-Hückel term is indeed large compared to the other terms which describe the deviations from the Debye-Hückel approximation. The method can be used to estimate the accuracy of the parameter values, in particular the charge number $Z_{\mathrm{E}}$, as determined by eq 7 and will be given in detail elsewhere (H.-J. Nolte and E. Neumann, unpublished results).

Data Analysis. Relaxation times were analyzed according to eq 3 with a second-order polynomial regression computer program introduced by Rosenberry \& Neumann (1977). Ionic strength dependencies were quantified according to eq 7 with a minimization function program (subroutine VAO4A, Harwell Subroutine Library) obtained from Dr. D. Lancet (Lancet \& Pecht, 1976). Error factors for parameters estimated from
Table I: Kinetic Parameters ${ }^{a}$ for the Interaction of $\mathrm{N}$-Methylacridinium with Acetylcholinesterase at Various Ionic Strengths

\begin{tabular}{ccccc}
$\begin{array}{c}\text { expt } \\
\text { no. }\end{array}$ & $\begin{array}{c}\text { ionic } \\
\text { strength } \\
(\mathrm{mM})\end{array}$ & $k_{12}\left(\mathrm{nM}^{-1} \mathrm{~s}^{-1}\right)$ & $K_{\mathrm{D}}(\mu \mathrm{M})$ & $k_{21}\left(\mathrm{~s}^{-1}\right)$ \\
\hline 1 & 1.37 & $6.38 \pm 0.21$ & 0.012 & 77 \\
2 & 4.54 & $4.52 \pm 0.07$ & 0.025 & 114 \\
3 & 13.5 & $3.40 \pm 0.07$ & 0.046 & 157 \\
4 & 13.5 & $3.57 \pm 0.07$ & 0.039 & 140 \\
5 & 26.9 & $2.10 \pm 0.04$ & 0.073 & 153 \\
6 & 39.7 & $1.58 \pm 0.02$ & 0.087 & 137 \\
7 & 75.9 & $1.11 \pm 0.02$ & 0.131 & 145 \\
8 & 121 & $0.89 \pm 0.02$ & 0.187 & 166 \\
9 & 121 & $0.80 \pm 0.03$ & 0.260 & 209 \\
\hline
\end{tabular}

${ }^{a}$ Determined at $25^{\circ} \mathrm{C}, \mathrm{pH} 7.1 \pm 0.2$, according to eq 3 and corrected for dilution and evaporation as outlined under Experimental Section. Values of $C_{\mathrm{E}}$ init calculated from eq 3 were 0.09 $\mu \mathrm{M}$ (expt 1), $0.17 \mu \mathrm{M}$ (expt 2), $0.61 \mu \mathrm{M}$ (expt 3-8), and $6.28 \mu \mathrm{M}$ (expt 9). Error estimates for $k_{12}$ were calculated by computer analysis, but error estimates for $K_{\mathrm{D}}$ and $k_{21}$ could not yet be calculated by the program [see Rosenberry \& Neumann (1977)].

eq 7 were obtained from a weighted least-squares analysis of the following linear form of eq 7 :

$$
\left[\frac{\Delta I_{\mathrm{c}}^{1 / 2}}{\Delta\left(\log k_{12}\right)}\right]^{1 / 2}=\frac{1+B \bar{a} \bar{I}_{\mathrm{c}}^{1 / 2}}{\left(2 A Z_{+} Z_{-}\right)^{1 / 2}}
$$

where each $\bar{I}_{\mathrm{c}}$ is the mean of $I_{\mathrm{c}}$ values associated with each $\Delta I_{\mathrm{c}}$.

Results

Ionic Strength Dependence of Relaxation Spectra Associated with $N$-Methylacridinium and Acetylcholinesterase. Relaxation measurements were conducted at $\mathrm{pH} 7$ during the course of a titration of a fixed amount of enzyme with increasing amounts of the fluorescent ligand in the temperature-jump cell. Analysis of the dependence of the single observed slow relaxation time $\tau$ on the total ligand concentration $C_{\mathrm{L}}{ }^{\text {tot }}$ gave simultaneous estimates of the total initial enzyme normality $C_{\mathrm{E}}{ }^{\text {init }}$, the equilibrium dissociation constant $K_{\mathrm{D}}$, and the bimolecular association rate constant $k_{12}$. Values of these estimates in experiments at various ionic strengths are given in Table I. In addition, the equilibrium fluorescence titration data also provided independent estimates of $C_{\mathrm{E}}{ }^{\text {init }}$ and $K_{\mathrm{D}}$. An indication of the agreement between the kinetic and equilibrium estimates is given by the average $C_{\mathrm{E}}{ }^{\text {init }}$ values calculated from experiments $3-8$ in Table I for which the input enzyme quantities were the same: the relaxation time analysis gave $0.61 \pm 0.02 \mu \mathrm{M}$, while the equilibrium analysis yielded 0.64 $\pm 0.05 \mu \mathrm{M}$.

Estimates of $k_{12}$ and $K_{\mathrm{D}}$ in Table I indicate that these parameters are highly dependent on ionic strength. This dependence of $k_{12}$ is shown graphically in Figure 1, where a plot based on eq 7 is presented. According to Scheme I, the dissociation rate constant $k_{21}$ is given by the product of $k_{12}$ and $K_{\mathrm{D}}$. Values of $k_{21}$ are also listed in Table I, and these values show much less dependence on ionic strength than $k_{12}$ values do. A slight tendency toward lower $k_{21}$ values is apparent at lower ionic strengths, but the statistical significance of this trend is questionable. The mean value of $k_{21}$ for experiments $2-9$ is $153 \pm 10 \mathrm{~s}^{-1}$. Thus, most of the ionic strength dependence of $K_{\mathrm{D}}$ is contributed by $k_{12}$.

Relaxation measurements were conducted at $\mathrm{pH} 7$ in contrast to the $\mathrm{pH} 8$ measurements reported earlier (Rosenberry \& Neumann, 1977) for two reasons: (1) the greater buffering capacity of $\mathrm{pH} 7$ phosphate better maintains the $\mathrm{pH}$ in the 


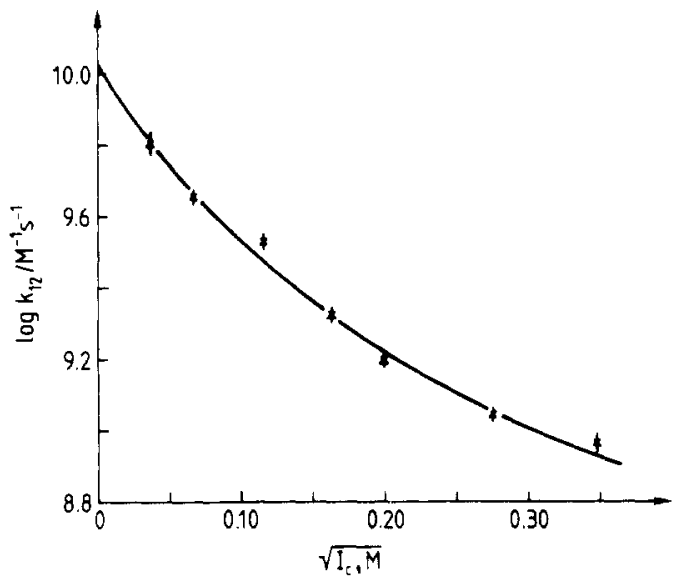

FIGURE 1: Dependence of the bimolecular association rate constant $k_{12}$ for $N$-methylacridinium and acetylcholinesterase on ionic strength at $25^{\circ} \mathrm{C}$. Values of $k_{12}$ are taken from Table I and plotted according to eq 7. The best fit line was calculated as noted under Experimental Section. The vertical bar through each point indicates twice the standard error.

lowest ionic strength experiments and (2) the progressive loss of ligand fluorescence during the titration, presumably due to the adsorption of the ligand on the quartz cell walls, is decreased. At equivalent $0.12 \mathrm{M}$ ionic strengths, the progressive loss of fluorescence at each $C_{\mathrm{L}}$ tot point in this study averaged $1 \%$ or about half that observed previously at $\mathrm{pH} 8$. However, the progressive loss at lower ionic strengths at $\mathrm{pH}$ 7 was greater and approached $3 \%$ per point in some experiments. Complete compensation for such progressive losses would alter $k_{12}$ and $k_{21}$ estimates by at most $10-15 \%$ [see Rosenberry \& Neumann (1977)]. Furthermore, the variability of the progressive loss called such compensation into question; for example, experiment 3 averaged a 1.0\% progressive loss while duplicate experiment 4 averaged a $2.5 \%$ progressive loss, yet the kinetic parameter estimates are similar. No compensation for progressive loss was made for estimates in Table I.

The experimental data in Table I were obtained at relatively low $C_{\mathrm{L}}{ }^{\text {tot }}$ values to maximize the accuracy of the kinetic parameter estimates involving the enzyme catalytic site. No consistent evidence of a second peripheral enzyme site for $\mathrm{N}$-methylacridinium was observed in either the equilibrium or the relaxation data. Tentative evidence consistent with such a site had been demonstrated with somewhat higher $C_{\mathrm{L}}{ }^{\text {tot }}$ points at $\mathrm{pH} 8$ in our earlier study. Examination of the slow relaxation amplitudes in this study revealed a linear dependence on $\bar{C}_{\mathrm{E}} \bar{C}_{\mathrm{L}} /\left(\bar{C}_{\mathrm{E}}+\bar{C}_{\mathrm{L}}+K_{\mathrm{D}}\right)$ as in the previous study, but the corresponding $\Delta H$ derived from this dependence was $18.0 \pm$ $1.7 \mathrm{~kJ} / \mathrm{mol}(4.3 \pm 0.4 \mathrm{kcal} / \mathrm{mol}$; average of six experiments $)$ or only one-half that reported previously. The discrepancy is probably due to an incorrectly labeled capacitor in the study of Rosenberry \& Neumann (1977). Very fast ligand relaxation step(s) associated with the presence of enzyme were also observed here in addition to the generally predominant slow relaxation. The time course of these fast $\operatorname{step}(\mathrm{s})(<5 \mu \mathrm{s})$ is beyond the resolution capacity of the temperature-jump method. The dependence of these fast relaxation amplitude(s), $\Delta F_{\text {fast }}$, on $\bar{C}_{\mathrm{L}}$ differed from that expected for ligand binding to a low-affinity peripheral site in that $\Delta F_{\text {fast }} / \bar{C}_{\mathrm{L}}$ decreased over about the same $\bar{C}_{\mathrm{L}}$ range as the corresponding ratio $\Delta F_{\text {slow }} / \bar{C}_{\mathrm{L}}$ for the slow relaxation. Thus, calculation of any coupling factor $\theta$ was precluded, and the possible correction of the slow relaxation times for ligand coupling to fast relaxations was resolved, as outlined under Experimental Section,

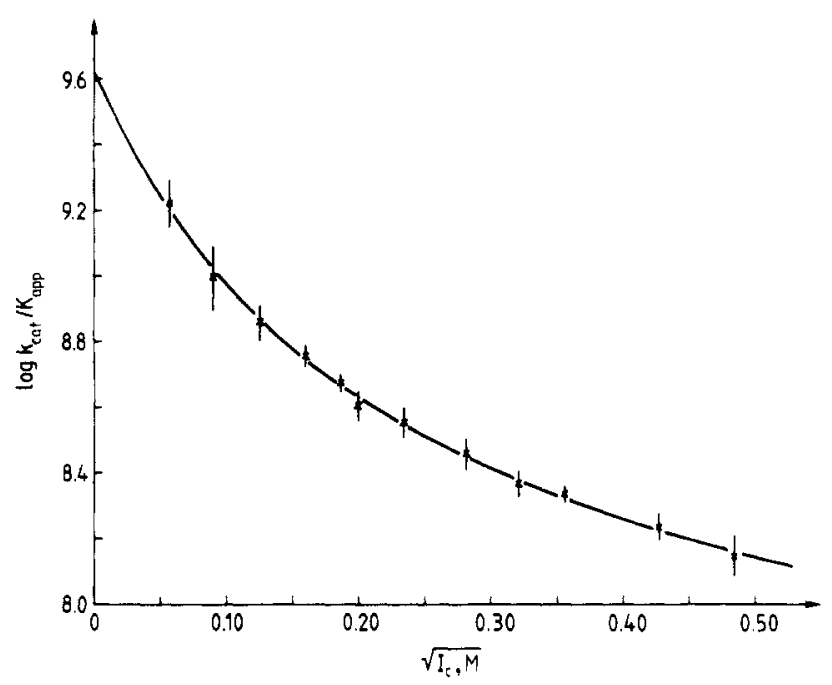

FIGURE 2: Dependence of the second-order hydrolysis rate constant $k_{\text {cat }} / K_{\text {apo }}$ for acetylthiocholine and acetylcholinesterase on ionic strength at $25^{\circ} \mathrm{C}$. Values of $k_{\text {cat }} / K_{\text {app }}$ were determined from eq 5 as outlined under Experimental Section at $\mathrm{pH}$ 6.8-7.1 and $C_{\mathrm{S}}=3.3 \mu \mathrm{M}$. The calculated line was determined as in Figure 1.

Table II: Kinetic Parameters for the Interaction of

Acetylthiocholine with Acetylcholinesterase at

Various Ionic Strengths

\begin{tabular}{rrrr}
\hline $\begin{array}{c}\text { ionic } \\
\text { strength } \\
(\mathrm{mM})\end{array}$ & $K_{\mathrm{app}^{a}(\mu \mathrm{M})}$ & $\begin{array}{c}k_{\text {cat }^{b}}{ }^{b} \\
\left(\mathrm{~s}^{-1} \times 0^{-4}\right)\end{array}$ & $\mathrm{p} K_{\mathrm{a}}$ \\
\hline 3.2 & $4.2 \pm 0.5$ & $0.69 \pm 0.09$ & $6.95 \pm 0.10$ \\
8.2 & $13.1 \pm 5.7$ & $1.29 \pm 0.58$ & $6.91 \pm 0.08$ \\
15.9 & $18.2 \pm 2.4$ & $1.30 \pm 0.19$ & $6.92 \pm 0.11$ \\
25.6 & $30.3 \pm 2.3$ & $1.73 \pm 0.14$ & $6.79 \pm 0.07$ \\
39.6 & $33.6 \pm 3.2$ & $1.34 \pm 0.13$ & $6.81 \pm 0.04$ \\
79.5 & $40.4 \pm 6.6$ & $1.16 \pm 0.20$ & $6.62 \pm 0.15$ \\
126.7 & $48.9 \pm 3.1$ & $1.06 \pm 0.07$ & $6.43 \pm 0.08$ \\
\hline
\end{tabular}

${ }^{a}$ Estimates were obtained from weighted reciprocal plots (Rosenberry \& Bernhard, 1971) according to eq 1 at pH 7.0 \pm 0.1 with assumption that $K_{\mathrm{a}} \simeq k_{3}{ }^{\prime} K_{\mathrm{a}}{ }^{\prime} / k_{\text {cat }} ; 25^{\circ} \mathrm{C}$. ${ }^{b}$ Calculated as the product of the corresponding $K_{\text {app }}$ in this table and $k_{\text {cat }} /$ $K_{\text {app }}$ in Figure 2.

by plotting $\tau^{-1}$ according to eq 2 . These plots were linear with $\theta=1$, and therefore coupling effects on the slow relaxation times were assumed to be negligible.

Ionic Strength Dependence of $k_{c a t} / K_{a p p}$ for Acetylthiocholine Hydrolysis by Acetylcholinesterase. The second-order hydrolysis rate constant $k_{\text {cat }} / K_{\text {app }}$ was determined under steady-state conditions at several ionic strengths. A graphical presentation of these data is given in Figure 2. Values of $k_{\text {cat }} / K_{\text {app }}$ increase at lower ionic strengths in a manner very similar to that shown earlier for $k_{12}$ with $N$-methylacridinium in Figure 1.

Estimates of $k_{\text {cat }} / K_{\text {app }}$ required small to moderate corrections for the ionic strength dependencies of $K_{\text {app }}$ and for $K_{\mathrm{a}}$, the equilibrium dissociation constant for a proton which inhibits the hydrolysis of positively charged ester substrates (see Scheme II and eq 1 and 5). As shown in Table II, the dependence of $K_{\text {app }}$ closely parallels the ionic strength dependence of $K_{\mathrm{D}}$ in Table I. In particular, $k_{\text {cat }}$, the product of $k_{\text {cat }} / K_{\text {app }}$ and $K_{\text {app }}$, shows evidence of only a slight ionic strength dependence except at the lowest ionic strength investigated. Thus, the ionic strength dependence of $K_{\text {app }}$ for acetylthiocholine, like that of $K_{\mathrm{D}}$ for $N$-methylacridinium, is contributed almost exclusively by the dependence of the bimolecular association rate constant for the interaction of the cationic ligand with the enzyme active site. The proton species associated with 


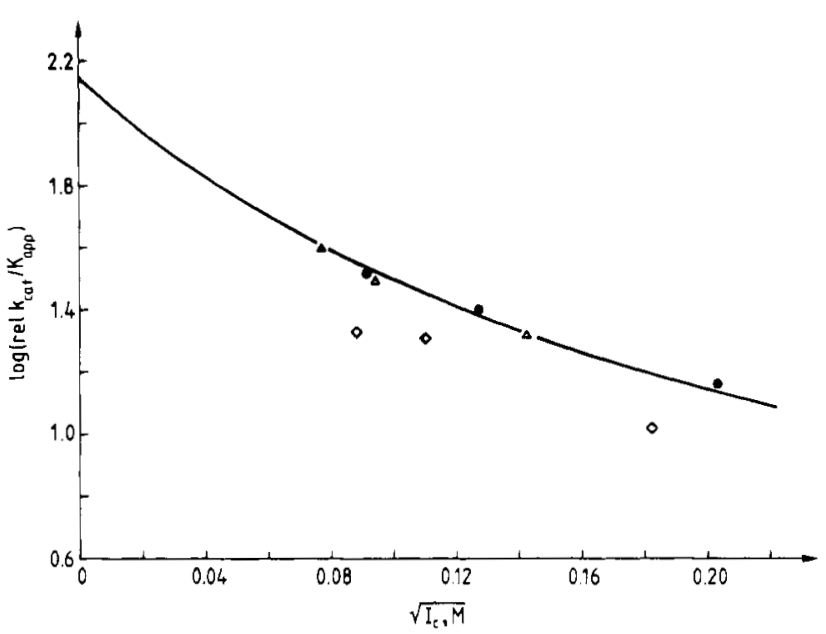

FIGURE 3: Comparison of dependence of relative $k_{\text {cat }} / K_{\text {app. }}$ on ionic strengths, $I_{\mathrm{c}}$, generated by three sodium salts and one calcium salt. Relative $k_{\text {cat }} / K_{\text {app }}$ is defined as $k_{\text {cat }} C_{\mathrm{E}}{ }^{\text {tot }} C_{\mathrm{S}} / K_{\text {app }}$ according to eq 5 , where $C_{\mathrm{S}}=3.3 \mu \mathrm{M}$ acetylthiocholine and $C_{\mathrm{E}}{ }^{\text {to }}$ was constant for all data points. Corrections for $K_{\mathrm{app}}$ and $K_{\mathrm{a}}$ were carried out as in Figure 2 according to eq 5 . The calculated line from Figure 2 was normalized to these data by means of a single control point in the assay solvent plus sodium phosphate, $\mathrm{pH} 6.93$, at $I_{\mathrm{c}}=3.2 \mathrm{mM}$. This sodium phosphate concentration was retained as a buffer for the other data points. These points and the respective additional $I_{\mathrm{c}}$ contributed by added salts were as follows: (๑) sodium chloride, 5.0 (pH 6.84), 13.0 ( $\mathrm{pH} 6.81)$, and $38.0 \mathrm{mM}(\mathrm{pH} \mathrm{6.76);}(\Delta)$ sodium citrate, $2.74(\mathrm{pH}$ $6.96), 5.55(\mathrm{pH} 7.05)$, and $16.9 \mathrm{mM}(\mathrm{pH} 7.15) ;(\diamond)$ calcium chloride, $4.5(\mathrm{pH} 6.87), 9.0(\mathrm{pH} \mathrm{6.76)}$, and $30.0 \mathrm{mM}(\mathrm{pH} \mathrm{6.58)}$.

$K_{\mathrm{a}}$ also appears to interact with the enzyme active site in an ionic strength dependent manner, as indicated by the $\mathrm{p} K_{\mathrm{a}}$ estimates in Table II. However, the 3.3-fold difference in $K_{\mathrm{a}}$ between the highest and lowest ionic strengths is less than the 10 -fold difference shown by $K_{\mathrm{app}}$; when $\mathrm{p} K_{\mathrm{a}}$ values are plotted against $I_{c}^{1 / 2}$, the dependence is linear within experimental error in contrast to the increase in slope observed for $k_{\text {cat }} / K_{\text {app }}$ in Figure 2 with increasing $I_{\mathrm{c}}{ }^{1 / 2}$. The difference is further considered under Discussion. Presumably the ionic strength dependence of $K_{\mathrm{a}}$ arises largely from the bimolecular association rate constant, although the rate constants comprising $K_{\mathrm{a}}$ have not yet been separated.

Relationship of Specific Ion Binding to the Ionic Strength Dependence. Despite the agreement between eq 7 and the experimental data in Figures 1 and 2, it could be argued that the apparent ionic strength dependence instead arises from specific $\mathrm{Na}^{+}$ion binding to the active site that partially competes with cationic ligands like $N$-methylacridinium, acetylthiocholine, and acetylcholine (H. R. Smissaert, unpublished results). This alternative mechanism was examined in Figure 3 by comparing the ionic strength dependence of relative $k_{\text {cat }} / K_{\text {app }}$ values in the presence of sodium chloride and sodium citrate with the calculated dependence from Figure 2 in the presence of sodium phosphate. The largely trivalent citrate ion contributes most of the ionic strength in sodium citrate solutions, and thus at a given ionic strength the $\mathrm{Na}^{+}$ concentration in a sodium citrate solution is only about half that in a sodium chloride solution. It is clear from Figure 3 that $k_{\text {cat }} / K_{\text {app }}$ follows the same ionic strength dependence for all three sodium salts and therefore that a specific $\mathrm{Na}^{+}$ion binding to the active site is not involved.

The ionic strength dependence of relative $k_{\text {cat }} / K_{\text {app }}$ in the presence of calcium chloride is also shown in Figure 3 and does not follow the dependence of the sodium salts. Values of $k_{\text {cat }} / K_{\text {app }}$ are some $30 \%$ lower at an equivalent ionic strength in calcium chloride. This suggests that a specific $\mathrm{Ca}^{2+}$ binding
Table III: Effective Enzyme Charge Number $Z_{\mathrm{E}}$ and the Mean Approach Distance $\bar{a}$ for the Interaction of Cationic Ligands with Acetylcholinesterase $^{\alpha}$

\begin{tabular}{lll}
\multicolumn{1}{c}{ ligand } & \multicolumn{1}{c}{$Z_{\mathrm{E}}$} & \multicolumn{1}{c}{$\bar{a}(\mathrm{~nm})$} \\
\hline$N$-methylacridinium & -6.3 & 0.91 \\
acetylthiocholine & -9.0 & 1.30 \\
& $-8.5 \pm 2.2^{b}$ & $1.16 \pm 0.29^{b}$ \\
proton & $-1.7 \pm 0.3$ & $(0)$ \\
\hline
\end{tabular}

${ }^{a}$ Values of $Z_{\mathrm{E}}$ and $\bar{a}$ were calculated from eq 7 with program VA04A (see Experimental Section) with the data for $k_{12}$ in Figure 1 for $N$-methylacridinium and for $k_{\text {cat }} / K_{\text {app }}$ in Figure 2 for acetylthiocholine. Values associated with the proton utilized $\mathrm{p} K_{\mathrm{a}}$ data in Table II plus three additional points (not shown) and result from a linear analysis according to eq 7 in which $\bar{a}$ is assumed equal to zero. ${ }^{b}$ Calculated with the same acetylthiocholine data as in footnote $a$ from eq 8 . Ten roughly equal $\Delta\left[\log \left(k_{\text {cat }} / K_{\text {app }}\right)\right]$ values were generated from the twelve experimental points by using no experimental point more than twice.

may interfere modestly with cationic ligand binding to the active site, particularly at low ionic strengths.

\section{Discussion}

High Bimolecular Association Rate Constants. Extrapolation to zero ionic strength provides estimates of $k_{12}{ }^{0}=1.1$ $\times 10^{10} \mathrm{M}^{-1} \mathrm{~s}^{-1}$ for $N$-methylacridinium in Figure 1 and of $\left(k_{\mathrm{cat}} / K_{\mathrm{app}}\right)^{0}=4.2 \times 10^{9} \mathrm{M}^{-1} \mathrm{~s}^{-1}$ for acetylthiocholine in Figure 2. This $k_{12}{ }^{0}$ value is the highest reported for the interaction of a small ligand with a specific protein binding site [see Hammes \& Schimmel (1971)], and in the following sections the electrostatic features that account for this high rate constant are examined from experimental and theoretical standpoints. These features also apply to acetylthiocholine (and presumably to acetylcholine) because $\left(k_{\text {cat }} / K_{\text {app }}\right)^{0}$ from the steady-state measurements appears closely analogous if not identical with a bimolecular association rate constant: $k_{\text {cat }} / K_{\text {app }}$ $\forall k_{\mathrm{s}} k_{2}{ }^{\prime} /\left(k_{\mathrm{ss}}+k_{2}{ }^{\prime}\right)$ (Scheme II). Also, previous evidence (Rosenberry, 1975a,b) has suggested that $k_{2}{ }^{\prime}>k_{-s}$ and thus that $k_{\text {cat }} / K_{\text {app }}$ is a close minimum estimate of the biomolecular association rate constant $k_{\mathrm{s}}$.

Apparent Charge on the Enzyme Active Site and the Ligand Approach Distance. The correspondence of the ionic strength dependence of the bimolecular association rate constants in Figures 1 and 2 to the Debye-Hückel relationship in eq 7 permits estimates of the net enzyme active site charge $Z_{\mathrm{E}} e_{0}$ and of the counterion approach distance $\vec{a} .^{1}$ These estimates, given in Table III, must be interpreted with care. The apparent active-site charge may well include a substantial contribution from the net charge on the overall enzyme [see Hammes \& Alberty (1959)]; the low acetylcholinesterase isoelectric point of 4.5 (Chen et al., 1974) suggests a considerable net negative charge at $\mathrm{pH} 7$. Furthermore, the possibility that the enzyme active site may be partially buried in a hydrophobic area of low dielectric constant [see Rosenberry (1975a)] could lead to an overestimate of $Z_{\mathrm{E}} e_{0}$, because both $A$ and $B$ in eq 7 are inversely proportional to the dielectric constant at the active-site surface. Thus, in our discussion of $Z_{\mathrm{E}}$ and $\bar{a}$ it should be understood that these are empirical parameters that reflect the overall interactions involving cationic ligands and the enzyme active site.

The most striking feature in Table III is the high effective charge on the enzyme active site with $N$-methylacridinium and

\footnotetext{
1 Because for acetylcholinesterase there is no evidence for polyvalent charged groups, $\left|Z_{\mathrm{E}}\right|$ is the effective number of monovalent anionic groups involved in the association of cationic ligands to the active site.
} 
Scheme III

$$
\mathrm{E}+\mathrm{L} \underset{k_{-1}}{\stackrel{k_{1}}{\rightleftharpoons}} \mathrm{E} \cdot \mathrm{L} \underset{k_{-2}}{\stackrel{k_{2}}{\rightleftharpoons}} \mathrm{EL}
$$

acetylthiocholine. Large values of $\left|Z_{\mathrm{E}}\right|$ and $\bar{a}$ contribute substantially to the high $k_{12}{ }^{0}$ estimate as noted below. It is unclear whether the difference in the $Z_{\mathrm{E}}$ or $\bar{a}$ estimates arising from these two ligands is statistically significant, because the VAO4A computational program unfortunately does not provide error estimates. Error estimates included in Table III for the alternative differential analysis (eq 8 ) may be somewhat higher than those expected from VAO4A because of the inherent scatter in a differential analysis. In addition to possible intrinsic differences in active-site characteristics toward the two ligands, differences might also arise from the nature of the rate constants measured. As noted in the previous paragraph, $k_{\text {cat }} / K_{\text {app }}$ appears to be closely related to but may not be identical with the association rate constant $k_{s}$. However, neither the measured dissociation rate constant $k_{21}$ (Table I) nor the unimolecular catalytic steps in $k_{\text {cat }}$ that involve the enzyme-substrate complex (Table II) showed large ionic strength dependencies, so it is likely that the dependence of $k_{\text {cat }} / K_{\text {app }}$ accurately reflects the dependence of $k_{\mathrm{s}}$. Another experimental difference between the $k_{12}$ and the $k_{\text {cat }} / K_{\text {app }}$ analyses is that no corrections for $K_{\mathrm{a}}$ were applied to the $k_{12}$ data, although such corrections do not appear to account for the differences in $Z_{\mathrm{E}}$ and $\bar{a}$ estimates. ${ }^{2}$

Table III indicates that $Z_{\mathrm{E}}$ for the enzyme active site is less toward the proton which acts as an active-site inhibitor than toward the other two cationic ligands. The precision of the $K_{\mathrm{a}}$ data was not sufficient to estimate an $\bar{a}$ value according to eq 7. Since $\mathrm{pH}$ changes alter the $Z_{\mathrm{E}}$ value of the enzyme active sites by neutralization of anionic groups, it is difficult to get complete $K_{\mathrm{a}}$ data [this effect may account for the nonlinear inhibition by $\mathrm{H}^{+}$below $\mathrm{pH} 6$ noted by Rosenberry $(1975 b)]$. Above $\mathrm{pH} \mathrm{6,} \mathrm{this} \mathrm{effect} \mathrm{was} \mathrm{not} \mathrm{major;} \mathrm{estimates}$ of five of the seven $\mathrm{p} K_{\mathrm{a}}$ values in Table II were averages of separate determinations in which $\mathrm{pH}_{1}$ (eq 4 ) was $\sim 6$ on the one hand and $\sim 7$ on the other, and the separate determinations agreed within their standard errors. Equilibrium $K_{\mathrm{a}}$ values in other protein systems have ionic strength dependencies which suggest $\bar{a}$ values of $\sim 0.3 \mathrm{~nm}$ [Shire et al, 1974; but see Beetlestone \& Irvine (1968)]. If $B \bar{a}=1$ is inserted into the expression for $\mathrm{p} K_{\mathrm{a}}$ corresponding to eq 7 , a $Z_{\mathrm{E}}$ value of $-2.4 \pm 0.2$ can be calculated from the same data utilized in Table III. It is not surprising that values of $Z_{\mathrm{E}}$ and $\bar{a}$ for the proton interaction are quite different from those for other cationic ligands. Although protons and cationic ligands compete for the active site, the protonation site is thought to be a histidine residue adjacent to the anionic group(s) in the active site (Krupka, 1966).

Binding of N-Methylacridinium to the Active Site Appears To Be Diffusion Controlled. For assessment of the question of diffusion control, the simple overall reaction in Scheme I must be expanded to Scheme III (Eigen, 1954). Scheme III

\footnotetext{
${ }^{2}$ It is difficult to analyze the coupled system $\mathrm{H}^{+}+\mathrm{EL} \rightleftharpoons \mathrm{E}+\mathrm{L}^{+}+$ $\mathrm{H}^{+} \rightleftharpoons \mathrm{EH}+\mathrm{L}^{+}$involving $N$-methylacridinium, because multiple relaxations are observed at low pH (Rosenberry \& Neumann, 1977: H,-J. Nolte and T. L. Rosenberry, unpublished results) that may preclude correction of $k_{12}$ by a simple factor of $1+C_{H} / K_{\mathrm{a}}$. The effects of this $K_{\mathrm{a}}$ correction on the acetylthiocholine data were assessed by analyzing the ionic strength dependence of $k_{\text {cat }} / K_{\text {apo }}$ prior to correction of $C_{\mathrm{H}} / K_{\mathrm{a}}$ in eq 5 . The $K_{\mathrm{a}}$ correction had little effect on $Z_{\mathrm{E}}$, decreased $\bar{a}$ by $\sim 30 \%$, and increased $\left(k_{\text {cal }} / K_{\text {app }}\right)^{0}$ by $60 \%$. Thus, this correction would not reconcile the differences between $Z_{\mathrm{E}}$ and $\bar{a}$ for $N$-methylacridinium and acetylthiocholine in Table III.
}

differs from Scheme I by inclusion of an intermediate, an initial diffusional encounter complex E.L (in which the original hydration shells of the individual reaction partners $\mathrm{E}$ and $\mathrm{L}$ may still be intact), prior to the chemical transformation to EL that results in fluorescence quenching. Our relaxation studies with $N$-methylacridinium show no evidence of a second relaxation with a characteristic dependence of time constant and amplitude on ligand concentration, and thus $E \cdot L$ must be present only in very low steady-state concentrations. In this case, the rate constants in Schemes I and III are related according to (Eigen, 1954)

$$
\begin{aligned}
& k_{12}=k_{1} k_{2} /\left(k_{-1}+k_{2}\right) \\
& k_{21}=k_{-1} k_{-2} /\left(k_{-1}+k_{2}\right)
\end{aligned}
$$

For the overall reaction to be diffusion controlled, the condition $k_{2}>k_{-1}$ must be met. In the Appendix, theoretical values for $k_{1}{ }^{0}$ and $k_{-1}{ }^{0}$, the zero ionic strength values of $k_{1}$ and $k_{-1}$, are calculated with the assumption of the $Z_{E}$ and $\bar{a}$ estimates for $N$-methylacridinium in Table III. The calculations yield $k_{1}{ }^{0}$ $=1.7 \times 10^{10} \mathrm{M}^{-1} \mathrm{~s}^{-1}$ and $k_{-1}{ }^{0}=7 \times 10^{7} \mathrm{~s}^{-1}$. From Figure 1 and Table II, the respective experimental estimates of $k_{12}{ }^{0}$ and $k_{21}{ }^{0}$ are $1.1 \times 10^{10} \mathrm{M}^{-1} \mathrm{~s}^{-1}$ and $1.5 \times 10^{2} \mathrm{~s}^{-1}$. Insertion of these four rate constant values in eq 9 and 10 indicates that $k_{2}{ }^{0} \geq 1 \times 10^{8} \mathrm{~s}^{-1}$ and $k_{-2}{ }^{0} \geq 1 \times 10^{2} \mathrm{~s}^{-1}$. Thus, $k_{2}{ }^{0} \geq k_{-1}{ }^{0}$, and the overall bimolecular reaction in Scheme $I$ is at least partially and perhaps completely under diffusion control.

Closer examination of eq $1 \mathrm{~A}$ and $2 \mathrm{~A}$ (see Appendix) reveals the contributions of the large negative $Z_{\mathrm{E}}$ to the observed rate constants $k_{12}$ and $k_{21}$. The major effect is to reduce $k_{-1}$ by the electrostatic potential factor $\phi_{-1}$ and thus to make the overall reaction diffusion controlled. The factor $\phi_{-1}$ is 1.0 if $E$ and $L$ have no charge, $\sim 0.7$ if $Z_{E}=-1$ and $Z_{L}=+1$, but 0.037 if $Z_{E}=-6.3$ and $Z_{L}=+1$. Thus, the large negative $Z_{E}$ reduces $k_{-1} 30$-fold to a value of $k_{-1} \leq k_{2}$ independent of ionic strength. According to eq 9 and 10 , this reduction in $k_{-1}$ results in a significant increase in $k_{12}$ and a somewhat smaller decrease in $k_{21}$. The lack of ionic strength effects on $k_{-1}$ is supported experimentally by the small ionic strength dependence of $k_{21}$ (Table I). The large negative $Z_{\mathrm{E}}$ also contributes directly to an increase in $k_{1}$ and hence to $k_{12}$, but this effect is only 4.9 -fold at zero ionic strength and, being ionic strength dependent, decreases to rather small values at physiological ionic strengths.

The comparatively large values of $k_{12}$ observed with $N$ methylacridinium led to our previous suggestion (Rosenberry \& Neumann, 1977) that an enzyme surface area larger than the ligand binding site itself was effective in "trapping" a cationic ligand in the encounter complex E.L. (Scheme III). This larger surface area might include peripheral anionic sites from which ligand would move to the active site by surface diffusion. The high negative $Z_{E}$ (as well as the comparatively large $\bar{a}$ ) values in Table III support this concept, because six to nine negatively charged enzyme groups would be expected to be dispersed over an enzyme surface area greater than the immediate catalytic site. Furthermore, the differences in $Z_{\mathrm{E}}$ and $\bar{a}$ among the cationic ligands in Table III suggest that ligands may differ in their relative rates of surface diffusion and thus in the area of surface over which trapping is effective. Further studies are required to extend and clarify differences in apparent electrostatic parameters among ligands.

\section{Acknowledgments}

The authors are grateful to Carol Mays for her careful technical assistance with the steady-state kinetic data and thank Dr. H. R. Smissaert, Laboratory for Research on In- 
secticides, Wageningen, Netherlands, for communicating his data prior to publication.

Appendix

The theoretical expression for $k_{1}$ in Scheme III (Eigen, 1954, 1974; Eigen et al., 1964; Debye, 1942) may be written as

$$
k_{1}=\frac{\Omega N}{1000}\left(D_{\mathrm{E}}+D_{\mathrm{L}}\right) d_{\mathrm{E} \cdot \mathrm{L}} \phi_{1}
$$

In eq $1 \mathrm{~A}, \Omega=2 \pi$ is the physically plausible value for the solid angle of diffusional approach, $N$ is the Loschmidt-Avogadro number $6.02 \times 10^{23} \mathrm{~mol}^{-1}, D_{\mathrm{E}}+D_{\mathrm{L}} \simeq D_{\mathrm{L}} \simeq 10^{-5} \mathrm{~cm}^{2} \mathrm{~s}^{-1}$ is the estimate for the sum of the diffusion constants of the enzyme and the ligand, $d_{\mathrm{E} \cdot \mathrm{L}}$ is the mean distance between the centers of the ligand and the active site in the encounter complex; the electrostatic potential factor $\phi_{1}=\phi_{1}^{0} f_{\mathrm{E}} f_{\mathrm{L}} / f^{*}$ (cf. eq 6), and at zero ionic strength $\phi_{1}=\phi_{1}{ }^{0} \equiv \varphi[\exp (\varphi)-1]^{-1}$, where $\varphi \equiv Z_{\mathrm{E}} Z_{\mathrm{L}} e_{0}{ }^{2} /\left(4 \pi \epsilon_{0} \epsilon k T d_{\mathrm{E} \cdot \mathrm{L}}\right)$. The term $\varphi$, the ratio between the electrostatic and thermal energy, includes the elementary charge $e_{0}\left(1.60 \times 10^{-19} \mathrm{C}\right)$, the permittivity constant $\epsilon_{0}\left(8.85 \times 10^{-12} \mathrm{C}^{2} \mathrm{~J}^{-1} \mathrm{~m}^{-1}\right)$, the dielectric constant of water $\epsilon(79)$, and the thermal energy $k T\left(4.12 \times 10^{-21} \mathrm{~J}\right.$ at $25^{\circ} \mathrm{C}$ ). Substitution of $Z_{\mathrm{E}}=-6.3$ and $d_{\mathrm{E} \cdot \mathrm{L}}=\bar{a}=0.91 \mathrm{~nm}$ from Table III gives $\varphi=-4.9, \phi_{1}{ }^{0}=4.9$, and $k_{1}{ }^{0}=1.7 \times 10^{10}$ $\mathbf{M}^{-1} \mathbf{s}^{-1}$.

Derived from a similar approach, the theoretical expression for $k_{-1}$ (Eigen, 1954) is

$$
k_{-1}=\frac{1.5}{d_{\mathrm{E} \cdot \mathrm{L}}^{2}}\left(D_{\mathrm{E}}+D_{\mathrm{L}}\right) \phi_{-1}
$$

In eq $2 \mathrm{~A}$ the electrostatic potential factor $\phi_{-1} \equiv \phi_{-1}{ }^{0} f_{\mathrm{E} \cdot \mathrm{L}} / f^{*}$; but since both the intermediate encounter complex and the activated complex are ion pairs, $f_{\mathrm{E} \cdot \mathrm{L}} \simeq f^{*}$ and $\phi_{-1} \simeq \phi_{-1}{ }^{0}$ at all ionic strengths [but see Eigen et al. (1964)], where $\phi_{-1}{ }^{0}$ $\equiv \varphi[1-\exp (-\varphi)]^{-1}$. With $\varphi=-4.9, \phi_{-1}{ }^{0}=0.037$ and $k_{-1}{ }^{0}=$ $7 \times 10^{7} \mathrm{~s}^{-1}$.

\section{References}

Bates, R. G., \& Pinching, G. D. (1949) J. Am. Chem. Soc. $71,1274-1283$.

Beetlestone, J. G., \& Irvine, D. H. (1968) J. Chem. Soc. A, 951-959.

Changeux, J.-P. (1966) Mol. Pharmacol. 2, 369-392.

Chen, Y. T., Rosenberry, T. L., \& Chang, H. W. (1974) Arch. Biochem. Biophys. 161, 479-487.

Debye, P. (1942) Trans. Electrochem. Soc. 82, 265-272.
Eigen, M. (1954) Z. Phys. Chem. (Wiesbaden) 1, 176-200. Eigen, M. (1974) in Quantum Statistical Mechanics in the Natural Sciences (Mintz, S. L., \& Widmayer, S. M., Eds.) pp 37-61, Plenum Press, New York.

Eigen, M., \& DeMaeyer, L. (1963) Tech. Org. Chem. 8 (Part 2), 895-1054.

Eigen, M., Kruse, W., Maass, G., \& DeMaeyer, L. (1964) Prog. React. Kinet. 2, 287-318.

Ellman, G. L., Courtney, K. D., Andres, V., Jr., \& Featherstone, R. M. (1961) Biochem. Pharmacol. 7, 88-95.

Hammes, G. G., \& Alberty, R. A. (1959) J. Phys. Chem. 63, 274-279.

Hammes, G. G., \& Schimmel, P. R. (1971) Enzymes, 3rd Ed. $2,67-114$.

Hillman, G. R., \& Mautner, H. G. (1970) Biochemistry 9, 2633-2638.

Kortüm, G. (1972) Electrochemistry, Verlag Chemie, Weinheim/Bergstr., Germany.

Krupka, R. M. (1966) Biochemistry 5, 1988-1998.

Lancet, D., \& Pecht, I. (1976) Proc. Natl. Acad. Sci. U.S.A. 73, 3549-3553.

Mooser, G., \& Sigman, D. S. (1974) Biochemistry 13, 2299-2307.

Neumann, E., Rosenberry, T. L., \& Chang, H. W. (1978) in Neuronal Information Transfer (Karlin, A., Tennyson, V. M., \& Vogel, H. J., Eds.) pp 183-210, Academic Press, New York.

Pyttel, R., \& Robinson, J. B. (1974) Can. J. Pharm. Sci. 9, 67-71.

Rice, S. A., \& Nagasawa, M. (1961) Polyelectrolyte Solutions, pp 142-146, Academic Press, New York.

Rigler, R., Rabl, C.-R., \& Jovin, T. M. (1974) Rev. Sci. Instrum. 45, 580-588.

Rosenberry, T. L. (1975a) Adv. Enzymol. Relat. Areas Mol. Biol. 43, 103-218.

Rosenberry, T. L. (1975b) Proc. Natl. Acad. Sci. U.S.A. 72, 3834-3838.

Rosenberry, T. L. (1979) Biophys. J. 26, 263-290.

Rosenberry, T. L., \& Bernhard, S. A. (1971) Biochemistry $10,4114-4120$.

Rosenberry, T. L., \& Neumann, E. (1977) Biochemistry 16, 3870-3878.

Scatchard, G. (1932) Chem. Rev. 10, 229-240.

Shire, S. J., Hanania, G. I. H., \& Gurd, F. R. N. (1974) Biochemistry 13, 2974-2979.

Smith, R. M., \& Alberty, R. A. (1956) J. Phys. Chem. 60, 180-184. 